\title{
A design of home smart nursing robot based on Raspberry $P i$
}

\author{
Yuqi Zhao, Yizhun Peng*, Jie Liu, Xiaowei Wu, Jikai Zhao \\ College of Electronic Information and Automation, \\ Tianjin University of Science and Technology, Tianjin, 300222, China; \\ E-mail:*pengyizhun@tust.edu.cn \\ www.tust.edu.cn
}

\begin{abstract}
This product is based on Bluetooth, WIFI and other wireless technologies, with the Raspberry Pi 4 as the core, to meet the health, safety and entertainment needs of the serviced users, mainly the elderly, while assisting the leisure and entertainment of users when traveling outdoors. A multi-functional escort robot designed for safety and other issues. It has many methods such as temperature and humidity monitoring, harmful gas monitoring, and noise monitoring to ensure the safety of users. At the same time, it also has TV projection, audio-visual entertainment touch screen interaction, mobile phone projection and other entertainment functions.

Keywords: Pension; artificial intelligence; entertainment; convenience; accompany; Internet of things; big data
\end{abstract}

\section{Introduction}

With the rapid increase of the elderly population, the issue of elderly care has become increasingly prominent, especially for the elderly in empty nests. It has become the norm for the elderly to live alone at home while their children are at work. At the same time, in recent years, the nursing industry such as "monthly sister" has not only experienced frequent problems but also increased industry prices. The home intelligent nursing robot is specially designed for the elderly. It has multiple functions such as health monitoring, smart medicine cabinet, housekeeping services, communication entertainment, abnormal situation alarm, critical situation handling, and action interaction. It is very suitable for the elderly living alone ${ }^{1}$.

This home care intelligent robot called the spouse, just like its name, is an intelligent robot specially designed and developed for the elderly living alone. In order to realize the function of accompany the elderly indoors and outdoors, it is based on wireless technologies such as Bluetooth and WIFI. To meet the entertainment needs and health and safety monitoring of the elderly, it can also solve the problems of the elderly in travel, such as loading, rest, health, etc., in all aspects.

\section{System overall design ideas \\ 2.1 Overall functional framework}

The system includes three components: an indoor body, a physical sign monitoring bracelet, and an outdoor body. It combines Bluetooth communication technology, network communication technology, Internet of Things technology, GPS positioning technology, and intelligent path planning technology. Among the three components, the physical sign monitoring bracelet can monitor the elderly's blood oxygen, pulse, heart rate and other physical sign data and GPS location in real time ${ }^{2}$, and realize two-way data exchange through Bluetooth and embedded systems. Through the wireless data network, indoor and outdoor body networking can be realized, and network entertainment resources can be obtained. The outdoor subject can integrate the camera, sensor, GPS coordinates of the bracelet and other information to realize intelligent path planning.

When the user is indoors, the physical sign monitoring bracelet will send the user's physical sign information to the indoor subject via Bluetooth, and calculate the user's health information after processing by the host system algorithm. When the system determines that the user's physical signs are abnormal, it will send the user's

(C) The 2021 International Conference on Artificial Life and Robotics (ICAROB2021), January 21 to 24, 2021 
physical signs to the settings through the network The first contact sends a warning carefully and calls 120 when necessary. At the same time, the indoor main body can remind the user to take medicine on time to prevent the elderly from forgetting. The indoor main body can also monitor whether the living environment is safe and issue an alarm in real time while working, so as to avoid the loss of life and property caused by accidents such as fire and gas leakage. The double safety protection of the user's body and living environment is realized; the indoor host is equipped with a large LCD screen specially designed for the elderly, which can be used for entertainment by the elderly without excessively adversely affecting the eyes ${ }^{3}$.

When the user is outdoors, the physical sign monitoring and GPS system work as usual, and the outdoor subject will follow the user, and all the user needs to do is act as usual. The outdoor part is box-shaped with storage space inside, and users can use it as a mobile basket. In addition, the box can be used as a seat for rest after fatigue. The outdoor part serves as a self-moving storage space, a movable seat, and can also achieve autonomous climbing of stairs through wheels with a peculiar mechanical structure. It greatly facilitates the lives of elderly people with limited mobility.

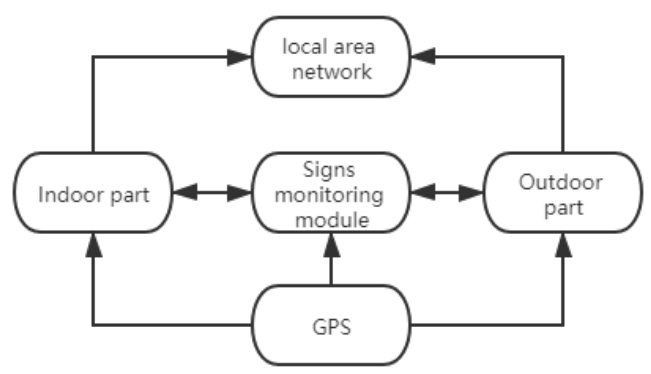

Fig.1 System Block Diagram]

\subsection{Vital Sign Monitoring Bracelet Design Scheme}

Body temperature detection: Because it is a bracelet to measure temperature, and according to the accuracy requirements, the method of contact temperature measurement is used to measure body temperature. The subsequent influence of the external temperature and the difference between the temperature of the wrist and the underarm temperature are calculated and compensated. So as to get a reliable body temperature. The LMT70 temperature sensor can be used as a low-power, highprecision sensor for temperature acquisition, and feedback to the microprocessor through analog-to-digital conversion. Further calculations are carried out by fitting compensation curves and calculation formulas of outdoor temperature, armpit temperature and wrist temperature in different environments
Blood pressure and heart rate monitoring: The photoelectric sensor can collect the pulse wave at our wrist. By analyzing the pulse wave's rising slope and band time and other characteristic parameters, a specific calculation formula can be obtained to estimate the blood pressure value. For example, when the blood pressure is high, the slope of the rising band of the ejection period will increase. This is also the method adopted by most bracelets on the market, because it is simple and convenient, and will not cause trouble to users ${ }^{4}$.

At the same time, the heart rate can be calculated through a certain algorithm. Nowadays, heart rate bracelets basically use light-sensing heart rate, which is tested by the principle of light reflection. Generally, they are affected by ambient light. Some products do not have heart rate data when facing strong light or completely black. Generally, green (OSRAM) is used with a photosensitive photodiode to detect the flow of blood flowing through the wrist at any time. When the user's heart beats, more blood will flow through the user's wrist, and the greater the amount of green light absorbed. Between the beating of the heart, blood flow is reduced, resulting in a reduction in the absorption of green light. The heart rate signal is effectively monitored and extracted through complex and sophisticated algorithms, most of which use the original NXP heart rate.

Based on the secondary development of the ready-made bracelet, integrated GPS module, easy to coordinate with the "wife" to complete positioning and tracking, built-in heartbeat detection, body temperature detection module, based on the Internet connection with the Raspberry Pi, collect the physical signs of the elderly at all times, by the Raspberry Pi Perform data review, and automatically send alarm and first aid information to children's mobile phones when physical signs of dangerous data appear ${ }^{5}$.

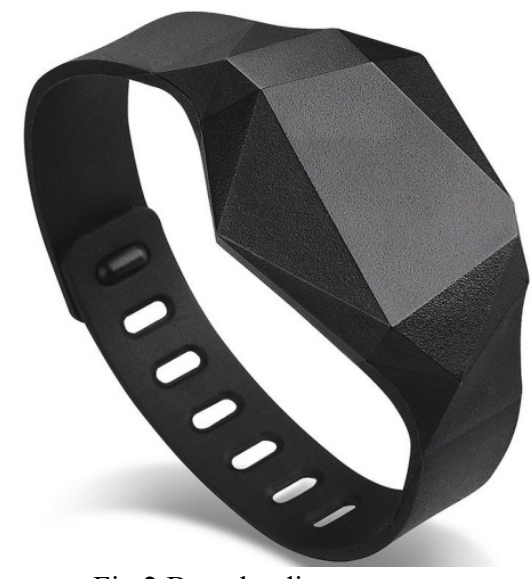

Fig.2 Bracelet diagram

\subsection{Interior design plan}

The product integrates the HR202 humidity detection

(C) The 2021 International Conference on Artificial Life and Robotics (ICAROB2021), January 21 to 24, 2021 
module and the DS18B20 temperature measurement module. By connecting the data to the core processor, it forms an important part of the indoor monitoring and alarm system.

The gas-sensitive material used in the MQ135 gas sensor is tin dioxide $(\mathrm{SnO} 2)$ with low conductivity in clean air. When there is pollutant gas in the environment where the sensor is located, the conductivity of the sensor increases with the increase in the concentration of pollutant gas in the air. Using a simple circuit, the change in conductivity can be converted into an output signal corresponding to the gas concentration. The MQ135 sensor has high sensitivity to ammonia, sulfide, and benzene vapors, and is ideal for detecting smoke and other harmful gases.

The MAX9814 high-sensitivity microphone sensor module integrated on the product can collect the sound in the environment and send it to the core processor, and then identify the abnormal sound in it, and then can judge the outdoor noise or the abnormal sound in the room (water leak, air leak, people Lock picking, etc.) and further processing as appropriate.

A unique sensor, the thermal imaging module IFD-X, is installed on the product. It monitors the temperature changes in the body of the elderly in real time and transmits the data to the Raspberry Pi for review. If data fluctuations are found to exceed the warning line, warning and detection information can be issued For children, on the other hand, it solves the physical changes that cannot be monitored by the bracelet, detects emergency situations one step in advance, and adds a stronger protection to the lives of the elderly.

IFD-X thermal imaging module, Red Eye Camera (hereinafter referred to as "REC" or "IFD-x" or "equipment") is a non-contact thermal imaging instrument based on infrared array high-precision temperature sensors and advanced software algorithms. Infrared imaging of any object within the field, the imaging resolution is up to $512 * 384$ pixels, the temperature sensitivity is $0.1^{\circ} \mathrm{C}$, the absolute accuracy is $\pm 1.5^{\circ} \mathrm{C}$, and the refresh frequency is up to $64 \mathrm{~Hz}$. Comes with storage and real-time clock, with real-time data output display, photo storage function, digital interface including UART and USB, can be directly connected to the computer and mobile phone, with the upper computer software or mobile phone APP program, it is very convenient to use $\mathrm{e}^{6}$.

This product is connected with a large-screen TV projection module, a touch screen interactive module, a mobile phone projection module, etc., which can meet various entertainment activities and external contacts in the daily life of the elderly. The details will not be repeated.

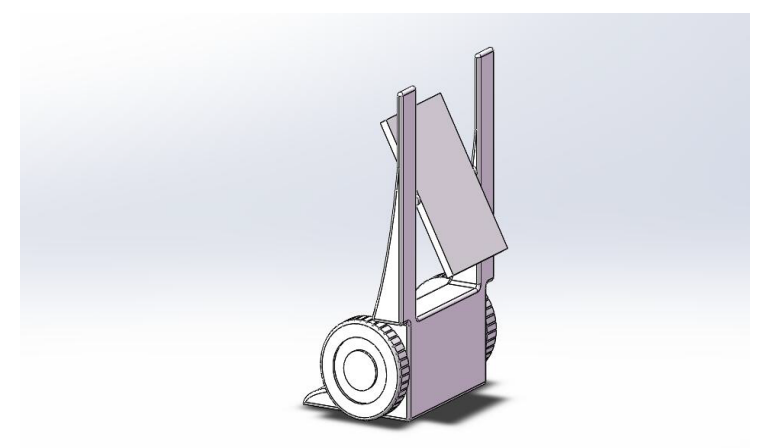

Fig. 3 Conceptual drawing of indoor part

\subsection{Outdoor part design scheme}

This product has the ability to exercise autonomously, and a large-capacity cabinet is reserved in the design, which can help the elderly transport vegetables when the user is out shopping, reducing the burden on the user and freeing hands. The product comes with a buffer module and a shock-proof module. Even if fragile dishes (such as eggs) are placed in the product, it will not break under most roads in the city. At the same time, the product is equipped with an auxiliary device for climbing stairs, which can assist users to climb stairs and reduce the difficulty of users carrying dishes upstairs. The materials are all made of high-quality compressive materials, and the design has a load-bearing structure. The maximum load-bearing capacity is $100 \mathrm{~kg}$. The user can sit directly on the product and rest. At the same time, the lid and handle are designed according to ergonomics. Even if sitting for a long time, there will be no back pain. At the same time, it will not exert any pressure on the inner basket. At the same time, the product also has a radio function for users to listen to and relax in their leisure time.

The content of intelligent path planning to achieve precise follow is as follows: The positioning method of our core design is based on visual positioning and sensor positioning, each has its advantages and disadvantages. The advantages of sensor positioning are:

1. Able to find the $x . y, z$ coordinates of the target

2. It can be positioned in 360 degrees

3. The positioning target is less affected by obstacles

Based on visual realization, the method is richer, and a variety of technology combinations can be used. For example, based on the depth camera to directly recognize the combination of human bones + face recognition + feature mark recognition. Atlas robots like Boston 
Dynamics are based on visual recognition to achieve walking and carrying objects. The advantages of visual recognition targets are: 1 . Not only can the x.y.z coordinates be calculated, but also the 3-dimensional deflection angle of the object relative to the camera. Can get richer decision-making information. 2. The visual unit can be used not only to identify targets, but also to identify most obstacles. In summary, we adopt a comprehensive positioning method of vision and sensing to obtain the best positioning effect. The sensor can be an infrared thermal imaging sensor, and the GPS function of the bracelet is used for positioning to determine the location of the owner. At the same time, three different positions on the product are installed with triangular 8266 modules. If the user chooses to use the mobile phone on his body, connect the three modules on the suitcase to this hotspot, and continuously obtain the current rssi value, and use this value to judge the distance between this module and the mobile phone. The three modules are sufficient to judge the mobile phone' $\mathrm{s}$ Direction, so as to achieve the purpose of following the user more accurately.

After the human body is recognized, the next step is generally to think of how to recognize the obstacle. Obstacle recognition, commonly used technologies include depth camera recognition, ultrasonic ranging, and infrared ranging. The advantages of depth camera and infrared ranging are that they are cheap and fast, but they cannot identify glass and black objects. Ultrasonic distance measurement can be used as a supplement. If the cost and size are not limited, lidar and millimeter multi-radar can also be considered. We use depth camera recognition, ultrasonic ranging, and infrared ranging to avoid obstacles.

Dynamic path planning, this product uses to build a 3D spatial map and rasterize the map into small grids that can or cannot be passed. With the aid of path planning algorithm, and then follow the target. The difficulty of the whole scheme is that the targets and robots are dynamic and need to be adjusted continuously to achieve optimal path planning. The camera is a more important part of the robot, this product uses the openmv highdefinition camera.

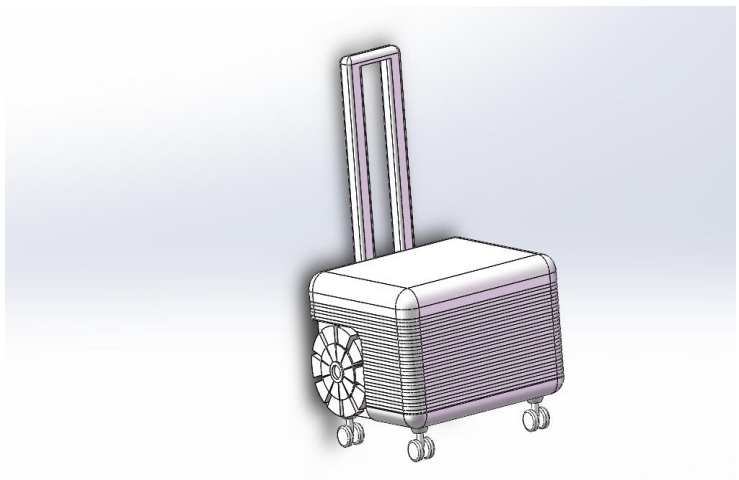

Fig.4 Concept map of outdoor part

\section{System software design \\ 3.1Software design of the interior part}

The software design of the indoor part includes three parts: the communication between the embedded device and the mobile phone client, the driving reading of the embedded device to various sensors ${ }^{8}$, and the communication between the embedded device and the physical sign monitoring bracelet.

1.Drive readings of various sensors by embedded devices:

The core processor of the embedded system adopts the stm32f103 series single-chip microcomputer of STM Company to realize the driving and reading of the sensor. The initialization of each sensor needs to be configured by the microcontroller according to different working principles. Sensor reading generally includes ADC reading and serial communication (including SPI, IIC, etc.).

2.The communication between the embedded device and the physical sign monitoring bracelet: send data via Bluetooth. Bluetooth $\mathrm{HC} 05$ is a master-slave Bluetooth serial port module. When the Bluetooth device is successfully paired and connected, the internal communication protocol of the Bluetooth can be ignored and the Bluetooth can be used as a serial port. When a connection is established, two devices share a channel, that is, the same serial port. One device sends data to the channel, and the other device can receive the data in the channel. hc-05 supports standard baud rates from 4800 bps to $1382400 \mathrm{bps}$. The baud rate must be matched when using it. Connect vec to 3.3 or $5 \mathrm{~V}$ GND to GND TX to RX on the microcontroller and RX to TX.

3.Feedback the user's health information to the mobile phone: 


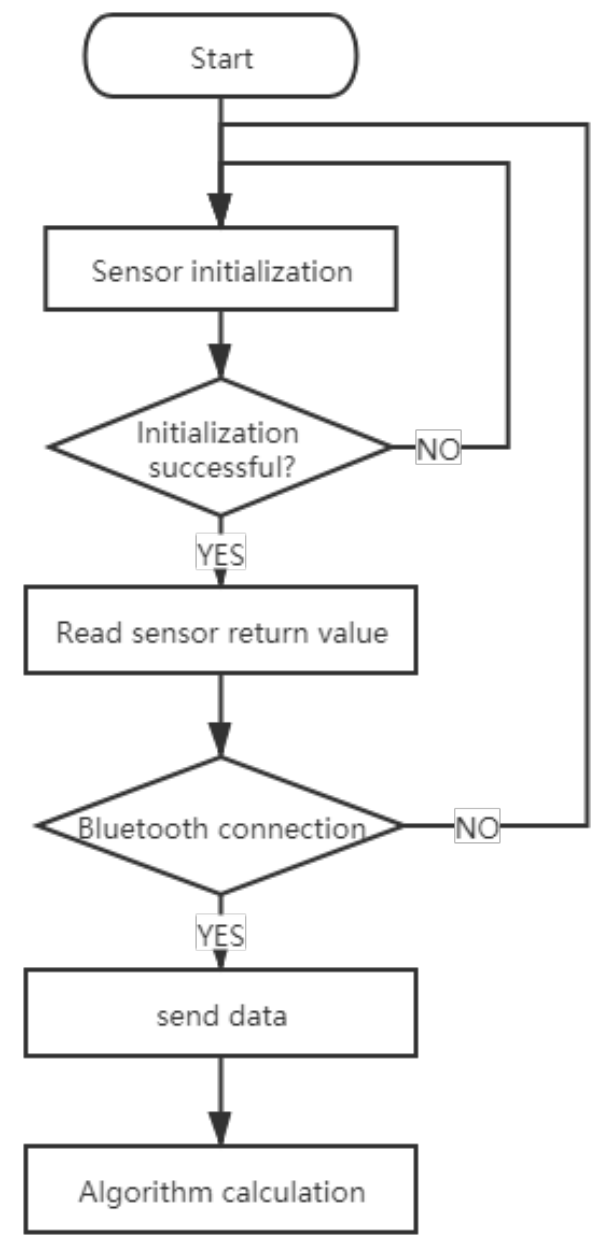

Fig.5 Feedback health information

\subsection{Sign detection software design}

1) Calculate the heart rate through a certain algorithm. The heart rate bracelet basically uses light-sensing heart rate. It is tested by the principle of light reflection. It is generally affected by ambient light. Some products are not exposed to strong light or all black. Heart rate bracelets with heart rate data are generally green (OSRAM) with a photosensitive photodiode to detect the flow of blood flowing through the wrist at any time. When the user's heart beats, more blood will flow through the user's wrist, and the greater the amount of green light absorbed. Between the beating of the heart, blood flow is reduced, resulting in a reduction in the absorption of green light. The heart rate signal is effectively monitored and extracted through complex and sophisticated algorithms, and the original phase heart rate of NXP is used.
2) MAX30100 is a sensor that can read heart rate and blood oxygen. The communication method is through IIC. Its working principle is to obtain the ADC value of the heart rate through infrared LED light irradiation. It is a non-invasive integrated pulse oximetry and heart rate monitoring sensor solution ${ }^{9}$.

\subsection{Outdoor software design}

There are many commonly used computer vision solutions, such as binocular vision, TOF-based depth cameras, and structured light-based depth cameras. The outdoor part of our current intelligent escort robot mainly works in an outdoor environment. The active light source will be greatly affected by conditions such as sunlight. Therefore, the passive vision solution of binocular vision is more suitable. Therefore, the vision solution we adopt is based on dual vision. Visual.

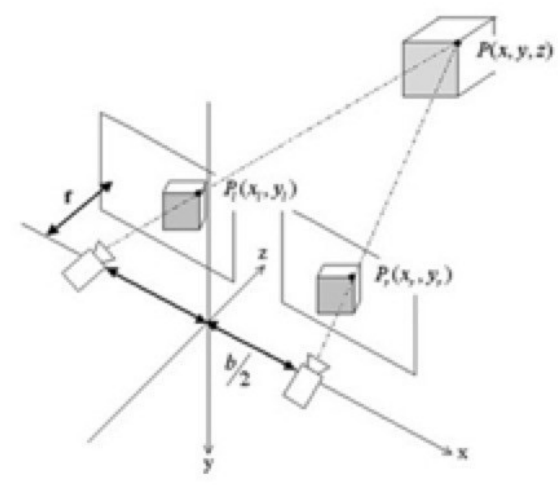

Fig.6 Binocular vision example image

The distance measurement of binocular vision is essentially a triangular distance measurement method. Because the positions of the two cameras are different, just like our two eyes, we see different objects. The same point $\mathrm{P}$ seen by the two cameras will have different pixel positions during imaging. At this time, the distance of this point can be measured by triangulation. Different from the structured light method, the points calculated by the structured light are actively emitted and known and determined, while the points calculated by the binocular algorithm are generally image features captured by the algorithm, such as SIFT or SURF features. The feature is calculated as a sparse image. In order to avoid obstacles well, we need to obtain a dense point cloud image and the depth information of the entire scene. This depth information can help us find walkable areas and obstacles in the map scene. The whole output is similar to the $3 \mathrm{D}$ point cloud image output by Lidar. 


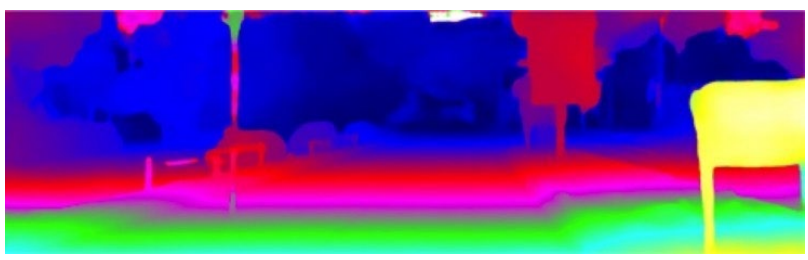

Fig.7 Actual output depth map, different colors represent different distances

At the same time, we use the vector field histogram (VFH) algorithm to achieve accurate obstacle avoidance and follow: during its execution, a local map based on polar coordinates is created for the current surrounding environment of the mobile robot. This local uses the grid map representation method, Will be updated by some recent sensor data. The polar coordinate histogram generated by the VFH algorithm is shown in the figure:

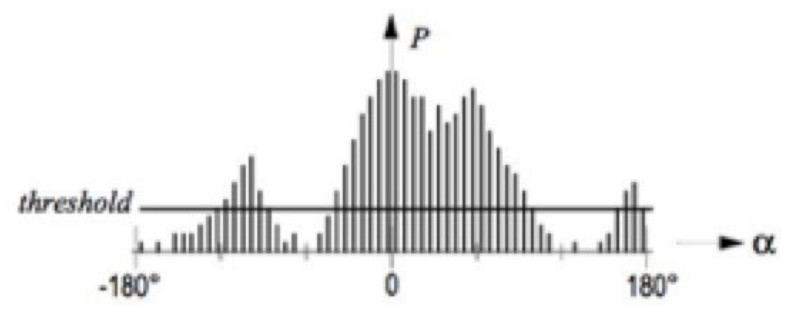

Fig.8 VFH algorithm polar coordinate histogram

In the figure, the $\mathrm{x}$-axis is the angle of the obstacle perceived by the robot as the center, and the y-axis indicates the probability $\mathrm{p}$ of the obstacle in this direction. In the actual application process, the histogram will first identify all gaps that are large enough to allow the robot to pass, and then calculate the cost function for all these gaps, and finally select the path with the lowest cost function to pass.

The cost function is affected by three factors: the target direction, the current direction of the robot, and the previously selected direction. The final generated cost is the weighted value of these three factors. The robot's selection preference can be adjusted by adjusting different weights. The VFH algorithm also has other extensions and improvements. For example, in the VFH+ algorithm, the limitations of robot kinematics are considered. Due to the difference in the actual underlying motion structure, the actual motion capability of the machine is limited. For example, the structure of a car cannot be turned in place at will. The VFH+ algorithm considers the blocking effect of obstacles on the trajectory of the robot's actual movement ability, and shields those trajectories that are not occupied by obstacles but cannot be actually reached because of their blocking. Our E-patrol robot uses a two-wheel differential drive motion form, which is very flexible, and its practical application is less affected by these factors. You can look at this icon for details:

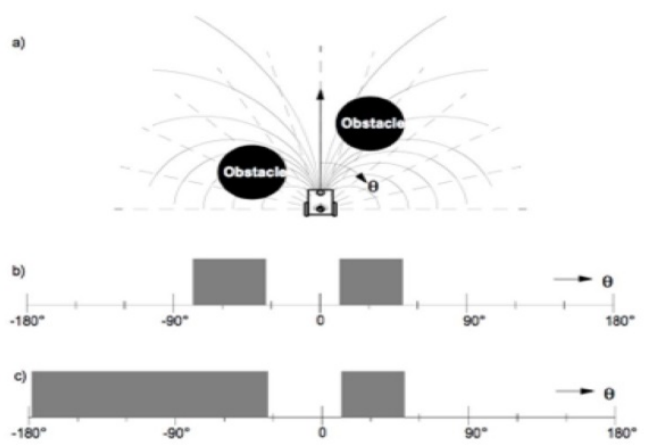

Fig.9 Influencing factors of cost function

\section{Concluding remarks}

Based on the serious aging problem in our country, the problem of elderly care has become increasingly prominent. The research and development of this robot fills the gap in the market, and to a certain extent alleviates the inconvenience of living alone for elderly people. It is a "wife" invented based on the design concept of serving the society and solving practical social problems. Innovative products of the times that are truly designed for the elderly. It is of great significance to solve social problems such as the difficulty of providing for the elderly in an aging society, the generally low quality of life of the elderly, and the unattended elderly in empty nests. With its comprehensive and humanized functions as its advantages, the home care intelligent escort robot will surely be recognized and supported by the society.

\section{Acknowledgements}

This research was supported by Student' s Platform for Innovation and Entrepreneur Training Program, the Ministry of education of China(202010057018).

\section{References}

1. Zhang Wenhui, Qi Naiming, Yin Hongliang. Adaptive neural variable structure robot trajectory tracking control [J]. Control and Decision. 2011 (04)

2. Yu Ting. Research on multi-robot queue curve movement [D]. Shanghai Jiaotong University 2009

3. Chen Canfeng. The principle and application of Bluetooth low energy technology $[\mathrm{M}]$. Beijing: Beihang University Press, 2013.

4. Shen Leixian, Pang Jiayi, Zhang Qingyun, Xu He. Design and implementation of positioning service platform based on Beidou navigation[J]. Computer Technology and Development, 2019, (01): 1-4. 
5. Chen Kaixuan, Xie Haitao. GPRS principle and its application[J]. Railway Communication Signal, 2003, 39(7): 7-8.

6. Wu Guojing, etc. Microcontroller Application Technology China Electric Power Press, 2004.1

7. Robot path planning Ren Chunmingdi, Zhang Jianxun1 (1. School of Information Technology and Science, Nankai University, Tianjin 300071; 2. Department of Information Science and Technology, Tianjin University of Finance and Economics, Tianjin 300222)

8. Human Health Index-Ling Yisheng [2] Wang Yongyan, Sun Yiwen

9. Wu Xiaofang. New Thoughts on Internet of Things and Big Data[J]. Communication World, 2017, (01): 12.[2017-08-31].

(C) The 2021 International Conference on Artificial Life and Robotics (ICAROB2021), January 21 to 24, 2021 